\title{
Concordancia de lesiones histológicas en ratones infectados por dos poblaciones de Trypanosoma cruzi de Chile
}

\author{
Tulio Pizzi P, Anne Wallace $\mathbf{R}^{\mathbf{a}}$, Rebeca Villagra $\mathbf{O}^{\mathbf{b}}$, \\ Sergio Muñoz V, Sylvia Ortiz Zc, Aldo Solari Id. \\ Prepatency, parasitemia and \\ histological lesions in mice infected \\ with two Trypanosoma cruzi \\ populations isolated in Chile
}

Background: The great variability in the clinical presentation of Chagas disease may depend in part in the genetic variability of Trypanosoma cruzi populations. Aim: To compare prepatent period, parasitemia, mortality and histological lesions in mice infected with two populations of Trypanosoma cruzi isolated in Chile. Material and methods: Two Trypanosoma cruzi populations, isolated from Chilean Triatomides and genetically characterized by kinetoplast restriction fragment DNA profiles, were compared. Two groups of $40 \mathrm{Balb} / \mathrm{c}$ mice were studied. Each mouse was inoculated with $10^{4}$ trypomastigotes, of the V-121 and sp COMB 2 Trypanosoma cruzi populations. The prepatent period, parasitemia, mortality and histopathological lesions, at different evolutionary stages of infection were registered during 32 days. Results: Prepatency and mortality were similar in both groups of mice. However, parasitemia was significantly greater in mice inoculated with V-121 than those inoculated with sp COMB 2. Amastigote pseudocysts and inflammation were present only in skeletal muscle and myocardium in both groups of mice. The intensity of tissue involvement was associated to the level of parasitemia, therefore it was greater in mice inoculated with V-121 population. Conclusions: V-121 population of Trypanosoma cruzi caused a greater parasitemia than COMB 2, in inoculated mice (Rev Méd Chile 2005; 133: 432-8).

(Key Words: Chagas disease; Protozoan infections)
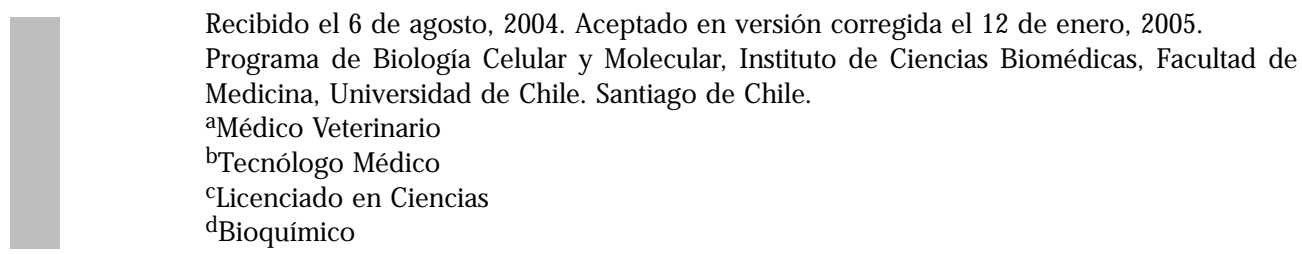

Correspondencia a: Aldo Solari I. Casilla 70086, Santiago,

Chile. E mail: asolari@med.uchile.cl 
L a enfermedad de Chagas afecta a más de veinte millones de personas en Latinoamérica y sólo en Chile se estiman alrededor de 150.000 personas infectadas con Trypanosoma cruzi, el agente causal de esta enfermedad ${ }^{1}$. Las razones de la gran variedad y gravedad de los cuadros clínicos que pueden presentarse durante las fases aguda y crónica, no están bien determinadas, aunque se han mencionado factores dependientes tanto del hospedero como del parásito ${ }^{2}$. Con relación a este último, la variabilidad genética de poblaciones de Trypanosoma cruzi ha sido establecida en base a sus características isoenzimáticas, lo que ha permitido su clasificación en dos linajes, $\mathrm{I} \mathrm{y} \mathrm{II}^{3} \mathrm{y}$ al tamaño de fragmentos de restricción del ADN de kinetoplasto ${ }^{4}$. Además, se han descrito en modelos experimentales, diferencias en el comportamiento biológico ${ }^{5}$ y en la respuesta a la lisis por complemento mediada por anticuerpos $^{6}$. El Trypanosoma cruzi es capaz de invadir prácticamente cualquier órgano o tejido, sin embargo, es ampliamente reconocido que algunas cepas presentan una mayor tendencia a ubicarse preferentemente en algunas localizaciones $^{7}$, siempre que ellas sean estudiadas bajo determinadas condiciones experimentales ${ }^{8}$. Por lo tanto, además de su mayor o menor grado de patogenicidad y virulencia, que pueden ser evaluadas por las curvas de parasitemia y las tasas de mortalidad ${ }^{2}$, la determinación de un particular histotropismo, podría ser un parámetro definitorio de carácter cualitativo de ciertas cepas de Trypanosoma cruzi. En el presente trabajo, como una contribución al conocimiento de poblaciones chilenas de Trypanosoma cruzi, aisladas de vectores de las regiones endémicas del país, se estudiaron algunas características biológicas y las lesiones histológicas, presentes en ratones infectados experimentalmente con dos poblaciones de Trypanosoma cruzi, provenientes de Triatoma infestans y de Mepraia spinolai, obtenidas en la II y IV regiones de Chile, respectivamente. El propósito fundamental fue estudiar comparativamente los registros de prepatencia, las curvas de parasitemia y la mortalidad con relación a las alteraciones histopatológicas en las distintas etapas evolutivas de la infestación y determinar el histotropismo de ambas poblaciones, las que corresponden al linaje I.

\section{MATERIAL Y MÉTODO}

Poblaciones de T cruzi. Se estudiaron dos poblaciones de $\mathrm{T}$ cruzi aisladas directamente de triatomineos. Una de ellas, V-121 obtenida de Triatoma infestans capturado en la II región de Chile y la segunda, sp COMB 2 proveniente de M spinolai de la IV región. Ambas poblaciones han sido clasificadas como pertenecientes al linaje I.

Ratones. La muestra estuvo constituida por un total de 80 ratones (40 machos y 40 hembras) $\mathrm{Balb} / \mathrm{c}$ de $18-20 \mathrm{~g}$ de peso, los cuales fueron separados en grupos de 40 individuos cada uno. Estos grupos fueron inoculados respectivamente con $10^{4}$ parásitos en $0,2 \mathrm{ml}$ de PBS provenientes de cada una de las dos poblaciones de $\mathrm{T}$ cruzi aisladas. Se registró la prepatencia y la mortalidad y se midió la parasitemia dos veces por semana durante 32 días $^{9}$. Grupos de animales fueron sacrificados a los 30, 60 y 150 días post inoculación, previa determinación de las cifras de parasitemia en el momento del sacrificio. Se obtuvieron para estudio histopatológico los siguientes órganos: cerebro, ganglios axilares, inguinales y mesentéricos, timo, pulmón, corazón, bazo, riñón, hígado, intestino delgado, piel y músculo esquelético. El material fue fijado en Bouin Hollande y teñido con hematoxilina-eosina.

Análisis de esquizodema. El kADN de los parásitos se obtuvo de acuerdo a condiciones previamente establecidas ${ }^{4}$. Los extractos de ADN kinetoplastídico fueron totalmente digeridos con un exceso de endonucleasa de restricción Eco RI siguiendo las indicaciones del proveedor. Los productos de la digestión fueron sometidos a electroforesis en una gradiente de gel de poliacrilamida de 4,5-10\% y posteriormente teñidos con nitrato de plata, según descripción anterior ${ }^{10}$.

Estadística. Los datos de parasitemia de las dos poblaciones de $\mathrm{T}$ cruzi fueron comparados por una prueba t de Student.

\section{RESULTADOS}

El comportamiento biológico de las dos poblaciones estudiadas fue: trece días de prepatencia para 
ambas poblaciones, con un promedio de $3.081,6 \times 10^{3}$ parásitos para la población V-121 y de $165,6 \times 10^{3}$ parásitos para la población sp COMB 2 y la mortalidad para V-121 fue de cinco ratones muertos de veinte ratones infectados y para sp COMB 2 cuatro ratones muertos de veinte infectados.

No se observó diferencias significativas entre ambas poblaciones con relación a la prepatencia y a la mortalidad. Sin embargo, las comparaciones de las medias de las parasitemias por medio de la prueba t de Student mostró diferencias significativas entre estas poblaciones ( $p<0,05$ ), presentando algunos animales inoculados con parásitos V-121, parasitemias máximas superiores a $3 \times 10^{6}$ parásitos por $\mathrm{ml}$, a diferencia de los animales inoculados con parásitos sp COMB 2, cuyas parasitemias máximas no alcanzaron a los $2 \times 10^{5}$ parásitos por $\mathrm{ml}$.

Los polimorfismos de fragmentos de restricción del kADN obtenidos después de la digestión con Eco RI permitieron distinguir a las poblaciones como diferentes entre sí.

La Figura 1 muestra los perfiles obtenidos para ambas poblaciones y se contrastan con otra población de $\mathrm{T}$ cruzi (V-124), perteneciente al linaje II.

El estudio histológico demostró la presencia de parásitos y de lesiones inflamatorias preferentemente en músculo esquelético y en menor proporción en miocardio auricular, característica que fue marcadamente similar en ambas poblaciones. La abundancia de nidos de formas amastigotas se asoció con la cuantía de la parasitemia presente en el momento del sacrificio (Tabla 1) y no con el tiempo de evolución de la infección. No hubo diferencias significativas entre machos y hembras con respecto a los parámetros biológicos medidos, sin embargo cabe mencionar la observación que los machos presentaron en general un mayor compromiso histológico.

La distribución de los focos parasitarios no fue uniforme, sino que mostró una tendencia a predominar en ciertas zonas de los sitios afectados, característica que fue especialmente notable en el músculo cardíaco, donde las lesiones comprometían casi exclusivamente al miocardio auricular (Figura 2). Es digno de destacar que los nidos parasitarios no presentaron una relación topográfica estricta con la distribución de la inflamación.
Por el contrario, con frecuencia se observaron nidos parasitarios sin inflamación adyacente (Figura 3). Los nidos fueron, en general, de tamaño mayor en músculo esquelético que en miocardio. Iguales diferencias se observaron en la extensión de la inflamación intersticial.

Las formas amastigotas contenidas en los nidos fueron de aspecto y tamaño variable, predominan-

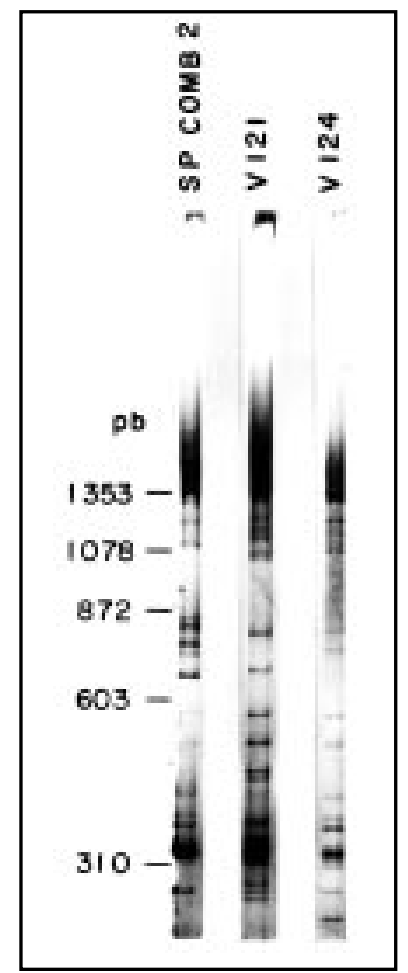

FIgURA 1. Perfiles de digestión de muestras de ADN kinetoplastídico de las poblaciones en estudio digeridas con Eco RI (V 121 y sp COMB 2) pertenecientes al linaje I, y una población perteneciente al linaje II de $\mathrm{T}$ cruzi ( $\mathrm{V}$ 124). Los marcadores de pesos moleculares aparecen en el borde izquierdo.

\section{Tabla 1. Promedios de parasitemias de ratones al} momento del sacrificio

\begin{tabular}{|c|c|c|}
\hline \multirow[t]{2}{*}{ Días } & \multicolumn{2}{|c|}{ Parásitos x 1033/ml } \\
\hline & V-121 & sp COMB 2 \\
\hline 30 & $5.563,6$ & 494,6 \\
\hline 60 & 204,7 & 49,7 \\
\hline 150 & 16,0 & 3,1 \\
\hline
\end{tabular}



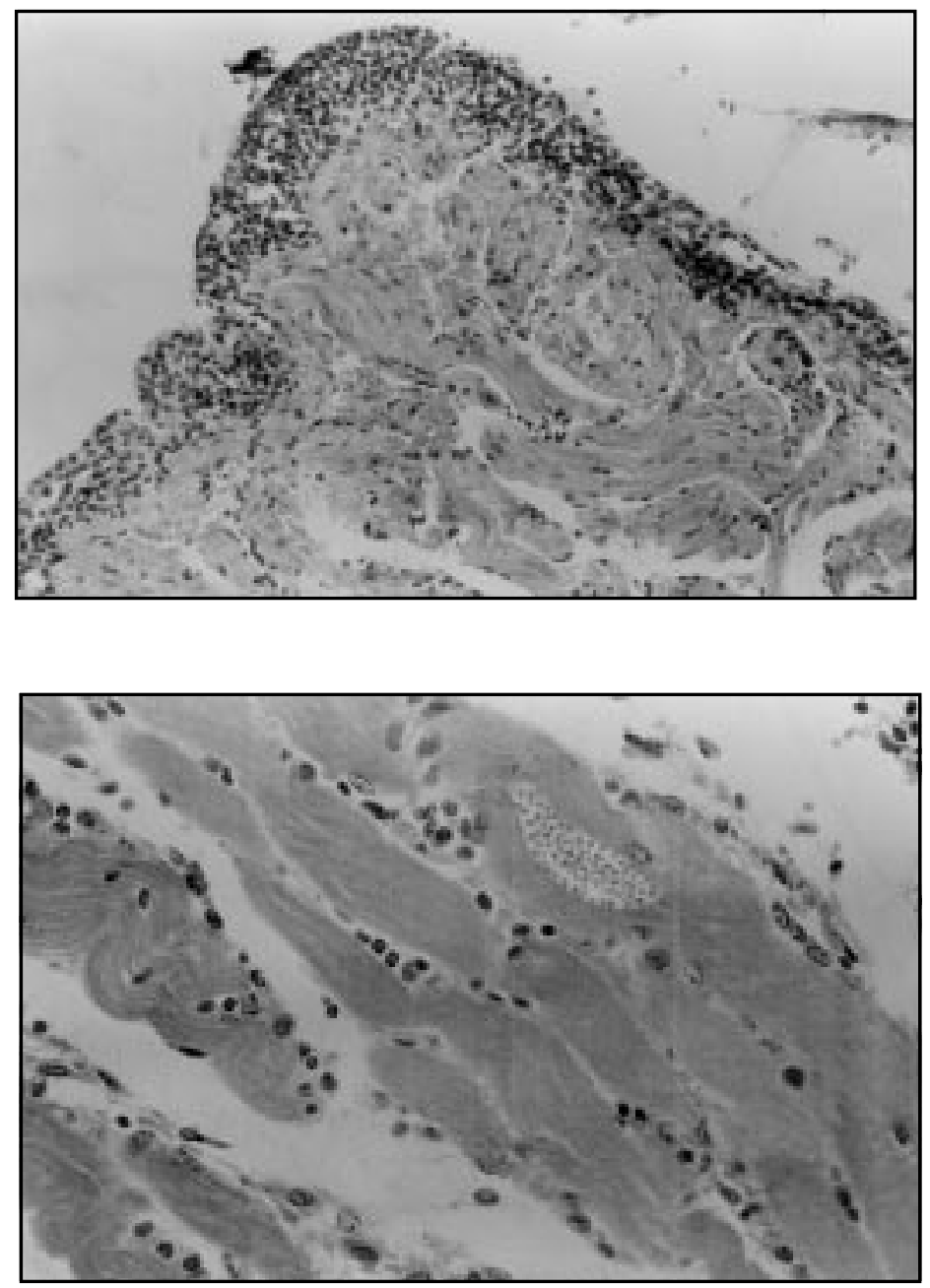

FIguRA 2. Microfotografía de corte de miocardio de un ratón infectado con formas tripomastigotas sanguíneas de la población de T cruzi V-121: Amplificación x 50. Obsérvese lesiones inflamatorias en miocardio auricular.

Figura 3. Microfotografía de corte de músculo esquelético de un ratón infectado con formas tripomastigotas sanguíneas de la población de $\mathrm{T}$ cruzi $\mathrm{sp}$ COMB 2: Amplificación x 160. Obsérvese un nido parasitario en músculo esquelético, sin inflamación adyacente. do formas redondeadas y pequeñas. En zonas con gran número de acúmulos parasitarios se observaron, ocasionalmente, nidos conteniendo formas de transición alargadas sugiriendo su transformación hacia tripomastigotes.

La inflamación intersticial en el músculo esquelético fue de variable intensidad y estuvo constituida preferentemente por infiltrados mononucleares predominantemente de linfocitos con menor proporción de monocitos, células plasmáticas y ocasionalmente neutrófilos y algunos eosinófilos (Figura 4).

Con frecuencia se observó áreas de necrosis segmentaria de las fibras musculares esqueléticas, las que aparecen como zonas con pérdida de la estriación y con coloración eosinofílica uniforme. En los casos de larga evolución, se presentaron segmentos de fibras musculares fibrosadas 0 en proceso de cicatrización $\mathrm{y}$, a veces, imágenes sugerentes de tentativas de regeneración con presencia de macrófagos y centralización y proliferación de núcleos sarcolemales (Figura 5).

Como ya se ha hecho notar, las lesiones miocárdicas fueron menos intensas y frecuentes que las de músculo esquelético. Su predominio fue auricular y se caracterizaron por la presencia de escasos y pequeños nidos de formas amastigotas, generalmente sin inflamación adyacente $\mathrm{y}$ focos inflamatorios discretos compuestos predominantemente por linfocitos y escasos polimorfo- 


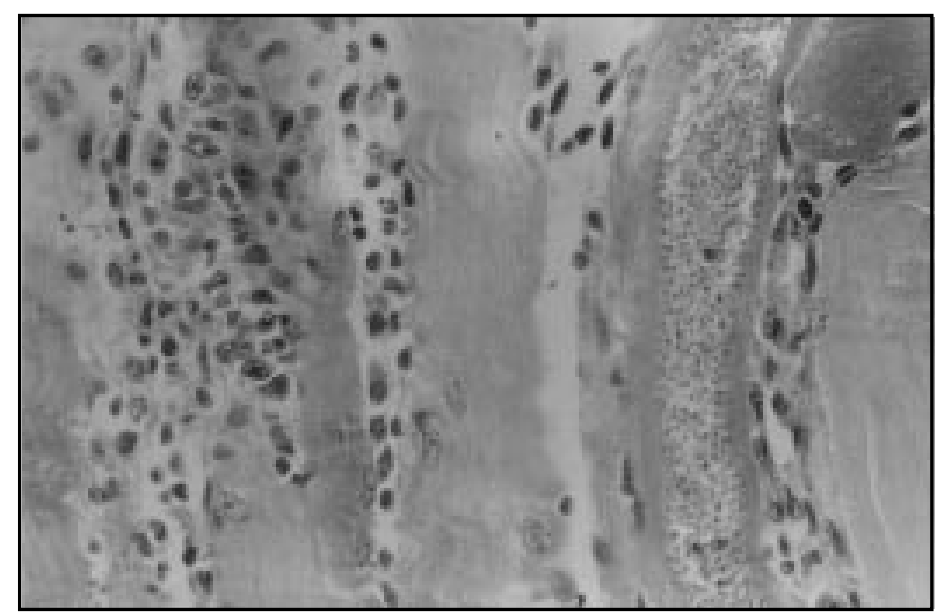

FiguRa 4. Microfotografía de corte de músculo esquelético de un ratón infectado con formas tripomastigotas sanguíneas de la población de T cruzi V-121: Amplificación x 160. Obsérvese nido parasitario en músculo esquelético $\mathrm{y}$ foco inflamatorio de predominio productivo, a distancia de la lesión parasitaria.

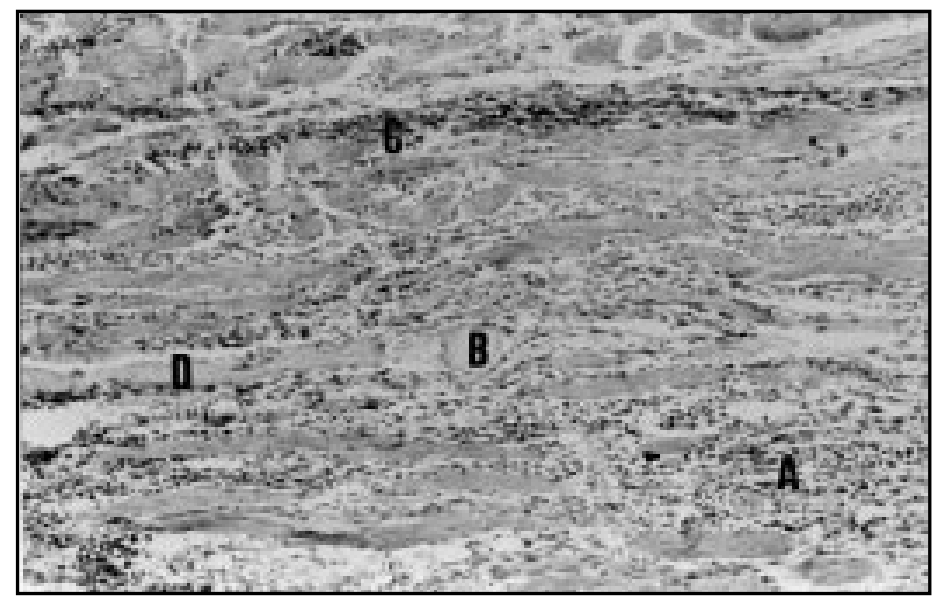

Figura 5. Microfotografía de corte de músculo esquelético de un ratón infectado con formas tripomastigotas sanguíneas de la población de T cruzi V-121: Amplificación x 50. Obsérvese extensa inflamación productiva intersticial en músculo esquelético (A), con algunas lesiones de necrosis de las fibras (B y D), y tentativas de reparación representadas por proliferación nuclear de las miocélulas (C). No se observa presencia de parásitos.

nucleares neutrófilos de ubicación preferentemente subendocárdica.

El resto de los órganos y tejidos examinados no reveló la presencia de parásitos ni lesiones inflamatorias en ninguno de los animales.

\section{Discusión}

El histotropismo de algunas poblaciones de $\mathrm{T}$ cruzi, ha sido ampliamente documentado desde los primeros trabajos experimentales $7,8,11,12$ y ha sido confirmado más recientemente por diversos investigadores $^{13-15}$. La determinación del tropismo de una cepa de $\mathrm{T}$ cruzi es un parámetro biológico que requiere de condiciones estrictamente controladas y que puede o no tener relación con las características clínicas de la enfermedad en las zonas geográficas de donde proviene $\mathrm{e}^{15,16}$. Sin embargo, podría, eventualmente, tener variaciones en el tiempo debido a presiones selectivas o según la forma en que es mantenida en diversos laboratorios, por ejemplo: número y cronología de los pasajes en cultivos o cepas de ratones en que es evaluada en animales de experimentación ${ }^{17,18}$.

Las dos poblaciones que estudiamos muestran en la actualidad un claro y definido miotropismo, sin que fuera posible encontrar nidos parasitarios en otros órganos o tejidos. Más aún, se observaron 
diferencias en la cuantía del parasitismo dentro del miocardio y el músculo esquelético, siendo mayor en este último. El tipo de compromiso preferente de cepas miotrópicas ya había sido señalado ${ }^{19}$. El predominio del compromiso esquelético sobre el miocardio también se observó en una cepa aislada en Méjico ${ }^{19}$.

La abundancia de los nidos fue variable y estuvo asociada con los niveles de la parasitemia en el momento del sacrificio de los animales.

Los infiltrados inflamatorios tuvieron similares características a las descritas por Molina y Kierzenbaum $^{20}$ en corazones humanos. En especial los infiltrados miocárdicos fueron parecidos al grupo HPII de su clasificación con un predominio linfocítico y escasos neutrófilos. En cambio la citología de las lesiones esqueléticas se asemejan más al tipo HPI de los mismos autores, en cuanto a la presencia ocasional de eosinófilos y un número algo mayor de neutrófilos. En estos infiltrados (pero no en los miocárdicos) pueden observarse también, en ocasiones, algunas células plasmáticas.

Es interesante confirmar la concordancia de los hallazgos histológicos en poblaciones provenientes de distintos transmisores y de zonas geográficas diversas cuyo único factor común es de pertenecer a un mismo linaje. El definido y exclusivo histotropismo hace posible especular

\section{REFERENCIAS}

1. Apt W, Aguilera X, Arribada A, Pérez C, Miranda C, SÁnchez G et AL. Treatment of chronic Chagas disease with itroconazole and allopurinol. Am J Trop Med Hyg 1998; 59: 133-8.

2. Walace A, Ortiz S, Sánchez G, Vilagra R, Puga M, Solari A. Studies on parasitemia courses and mortality in mice infected with genetically distant Trypanosoma cruzi clonets display dissimilar parasitemia courses. Biol Res 2001; 34: 83-90.

3. Barnabe $C$, Neubauer $K$, Solari A, Tibayrenc $M$. Trypanosoma cruzi: presence of the two major phylogenetic lineages and of several lesser discrete typing units (DTUs) in Chile and Paraguay. Acta Tropica 2001; 78: 127-37.

4. Venegas J, Ortiz S, Muñoz S, Solari A. Molecular karyotype and schizodeme analyses of $\mathrm{T}$ cruzi que ambas poblaciones pudieron haber tenido un origen común con una dispersión geográfica ulterior. Estos resultados se contrastan con los obtenidos anteriormente con la cepa Tulahuén, la cual pertenece al linaje II y que presenta un comportamiento biológico muy distinto al observado aquí con dos poblaciones de linaje I, ya que la primera adaptada en el modelo murino es de histotropismo reticulotrópico ${ }^{7,8}$. Además, la cepa Tulahuén difiere de las poblaciones V121 y sp COMB 2 por su mayor virulencia, expresada por una menor prepatencia, por más altas parasitemias y por la mortalidad total de ratones isogénicos en un promedio de 10 días y un máximo de 13 días ${ }^{11}$. El carácter marcadamente reticulotrópico de la cepa Tulahuén no excluye un escaso compromiso del músculo esquelético y cardíaco. No sabemos qué factores influyen en el histotropismo de $\mathrm{T}$ cruzi, pero es posible que ello dependa de la expresión de diversos receptores de superficie de las formas tripomastigotas.

Cabe señalar finalmente, que las poblaciones V-121 y sp COMB 2 no son cepas estabilizadas, sino poblaciones recientemente aisladas, por lo cual el pasaje seriado en un mismo huésped isogénico podría determinar cambios ulteriores en sus características biológicas.

stocks from Chilean triatomines. Parasitology 1997; 115: 41-6.

5. Solari A, Wallace A, Ortiz S, Venegas J, Sánchez G. Biological characterization of Trypanosoma cruzi stocks from Chilean insect vectors. Exp Parasitol 1998; 89: 312-22.

6. Waldace A, Sánchez G, Venegas J, Solari A. Lack of reactivity of lytic antibodies with bloodstream forms of Trypanosoma cruzi zymodemes generated in a mouse experimental model. Exp Parasitol 1995; 80: 176-85.

7. Pizzi T. Inmunología de la enfermedad de Chagas. Monografía Biológica 1957; U de Chile, Imp Stanley.

8. TALAFERRo WH, PIzZI T. Connective tissue reactions in normal and immunized mice to a reticulotropic strain of Trypanosoma cruzi. J Inf Dis 1955; 96: 199-226. 
9. BRENER Z. Therapeutic activity and criterion of cure on mice experimentally infected with Trypanosoma cruzi. Rev Inst Med Trop Sao Paulo 1962; 4: 389-96.

10. Goncalves A, Nehme N, Morel CM. An improved silver staining procedure for schizodeme analyses in polyacrylamide gradient gel. Mem Inst Oswaldo Cruz 1990; 85: 101-6.

11. Pizzi T, Prager R. Estabilización de la virulencia de una cepa de Trypanosoma cruzi por pasaje seriado en ratones de constitución genética uniforme: Análisis cuantitativo del curso de la infección. Biológica (Chile) 1952; 16: 3-12.

12. GALARD $H$. The variety of the tropism of different strains of Trypanosoma cruzi in the mouse. Prog Prot Excerpta Med Found (Amsterdam) 1965; 143-4.

13. De Scorza C, Urdaneta-Moraies S, Sampson-Ward L Urban Trypanosoma (Schizotrypanum) cruzi: pathology in white mice of isolates from Panstrongylus geniculatus. Ann Soc Belg Med Trop 1989; 69: 283-9.

14. Nunes MP, Sobral AC, CoutinHo SG. Quantification of Trypanosoma cruzi in the heart, lymph nodes and liver of experimentally infected mice, using limiting dilution analysis. Mem Inst Oswaldo Cruz 1992; 87: 503-9.
15. Oliveira EC, Stefani MM, Luquetti AD, Vencio EF, Mouna MA, SouZa C ET AL. Trypanosoma cruzi experimental Chagas disease: characterization of an isolate from a patient with associated digestive and cardiac form. Rev Soc Bras Med Trop 1993; 26: 25-53.

16. WatKins R. Comparison of infection produced by two strains of Trypanosoma cruzi in mice. J Parasitol 1966; 52: 958-61.

17. Perri P, De Diego J, Del Rey J, Mayer R, Gamallo C. Acute chagasic cardiopathy produced by a strain of Trypanosoma cruzi (tipo 1) in an experimental model. Ann Parasitol Hum Comp ; 65: 208-13. año ??

18. De Diego J, Penin P, Del Rey J, Mayer R, Gamalo C. A comparative pathological study of the strains of Trypanosoma cruzi in an experimental model. Histol Histopathol 1991; 6: 199-206.

19. BICE BE, ZELEDON R. Comparison of infectivity of strains of Trypanosoma cruzi: (Chagas 1909). J Parasitol 1970; 56: 663-70.

20. Molna HA, Kierszenbaum F. A study of human miocardial tissue in Chagas disease: distribution and frequency of inflammatory cell types. Int J Parasitol 1987; 17: 1297-305.

Agradecimientos

A la señora Gladys Piña por su colaboración en la preparación de las placas histológicas. 